\title{
Cognitive Function In Strategic And Non-Strategic Cerebral Infarction Locations, And White Matter Changes On Head MSCT Scan
}

\author{
Pinkan Lestari Kalalembang ${ }^{\mathrm{a}}$, Widiana Ferriastuti ${ }^{\mathrm{b}}$, Bambang Soeprijanto ${ }^{\mathrm{b}}$ \\ apinkankalalembang@gmail.com, ${ }^{b}$ widiana ferriastuti@yahoo.com \\ ${ }^{a}$ Resident, Department of Radiology, Faculty of Medicine Airlangga University, Dr. Soetomo General Academic Hospital Surabaya, \\ Indonesia \\ ${ }^{b}$ Radiologist, Department of Radiology, Faculty of Medicine Airlangga University, Dr. Soetomo General Hospital Surabaya, \\ Indonesia
}

\begin{abstract}
Stroke is one of leading cause of mortality. Stroke increases 5-8 times the risk of cognitive impairment. The presence of strategic infarct locations and white matter changes were reported to be associated with cognitive impairment on ischemic stroke. This study aims to describe the characteristic of cognitive function of cerebral infarction patient in strategic and non-strategic locations; and white matter changes. This is descriptive study with retrospective design. Data of ischemic stroke patient at the Diagnostic Radiology Installation of Dr. Soetomo Surabaya during January February 2020, were collected. Blennow scale was used to evaluate white matter changes, based on MSCT Scan. Degree of cognitive function assessed with MoCA-INA score. Total of 49 ischemic stroke patients, mostly in the age group of 51-60 and $>60$ years old (38,8\%), male (59,2\%), with strategic infarct locations $(75,5 \%)$, and Blennow scale 2-3 (55,1\%). Most of the subjects have decreased cognitive function (83.7\%). Subjects who experienced cognitive dysfunction were mostly found in the age group $41-50$ and $>60$ years old (34.7\%). Cognitive impairment were mostly found in male patients (46.9\%), with strategic infarct locations (69.4\%), and with Blennow scale $2-3(51 \%)$. Cognitive function in patient with strategic infarct locations, and white matter changes, mostly impaired.
\end{abstract}

Published by IJRP.ORG. Selection and/or peer-review under responsibility of International Journal of Research Publications (IJRP.ORG)

Keywords: cerebral infarction; cognitive function; white matter changes

\section{Background}

Stroke is the third leading cause of death after heart disease and cancer. In United Stated, there are 700,000 cases of stroke each year, 600,000 of them are ischemic strokes and 100,000 hemorrhagic strokes. ${ }^{1}$ Indonesia's Basic Health Research (Riset Kesehatan Dasar/ Riskesdas) in 2013 shows that stroke is the first cause of death in Indonesia. The prevalence increased from 8.2 per 1000 in 2007 to 12.1 per 1000 populations in $2013 .^{2}$ 
Cerebral infarction is a brain function disorder characterized by a sudden focal or global neurological deficit lasting more than 24 hours due to decreased blood flow. Cerebral infarction can occur in strategic locations or functionally important brain areas (internal capsule crus anterior - genu, thalamus, caudate nucleus, lentiform nucleus, basal frontal and carotid artery watershed areas. ${ }^{3}$

Globally, the morbidity due to ischemic stroke is almost 4 times greater than that of hemorrhagic stroke, where $25-30 \%$ of ischemic stroke lead to direct or delayed vascular cognitive impairment. ${ }^{4}$ Stroke increases 5-8 times the risk of cognitive dysfunction. ${ }^{5}$ Data from the Indonesian Stroke Registry in 2013 showed that $60.59 \%$ of stroke patients had cognitive impairments. The incidence of cognitive dysfunction in ischemic stroke patients was $60.44 \%$ at Hasan Sadikin Hospital Bandung in 2018. Memory is the most disturbed domain, which is occured in $75.82 \%$ cases. $^{6}$

Several predictors for post-stroke cognitive impairment, including acute cortical infarction, white matter changes, lacunar infarction, intracranial large vessel stenosis and educational level. ${ }^{7,8}$ The presence of infarction at strategic locations and White matter changes alone are reported to predict the occurrence of post-stroke cognitive impairment. ${ }^{9}$ Whereas Kliper et al reported that mild to moderate stroke patients with preexisting white matter changes were more susceptible to cognitive impairment, apart from new ischemic lesions. ${ }^{10}$ Data reported by the Framingham Heart Study in 2010 stated that extensive white matter changes in stroke patients doubled the risk of mortality and 4 times the risk of dementia. ${ }^{11}$

The combination of neuropsychological and imaging tests can be advantageous in the early detection of dementia and cognitive impairment. The assessment of cognitive impairment with the Montreal Cognitive Assessment (MoCA) score is more sensitive than Mini Mental State Examination (MMSE) and easy to use for the evaluation of post-stroke cognitive impairment in the acute stroke. ${ }^{12,13}$ Research by Wattjes et al reported that Multislice Spiral Computed Tomography (MSCT) head scan with 64 slices is a suitable and accurate imaging method for evaluating WMC which is almost equivalent to Magnetic Resonance Imaging (MRI). ${ }^{14}$

Thus it is important to be able to identify the presence of vascular cognitive impairment in ischemic stroke patients from an early stage, related to strategic and non-strategic infarction locations, and in the white matter changes in order to provide information for early cognitive rehabilitation.

\section{Methods}

\subsection{Study design and setting}

This is a descriptive study with a retrospective design. The research was conducted at the Diagnostic Radiology Installation of Dr. Soetomo General Hospital Surabaya during January - February 2020. The datas were collected from ischemic stroke patients who came to the emergency unit, and were hospitalized at Dr. Soetomo General Hospital Surabaya.

\subsection{Study population and sampling strategy}

The subjects of the study were patients with stroke infarction, who underwent an MSCT scan of the head without contrast at Dr. Soetomo General Hospital during January - February 2020 who met the inclusion criteria. Subjects were taken using consecutive sampling. The inclusion criteria in this study were: (1) Age over 21 years; (2) Stroke infarction which is diagnosed from clinical and additional examination by Neurologist; (3) Showed of the infarction on the head MSCT scan without contrast; (4) Patients with 
stroke infarction who were examined for the MoCA-Ina score by Neurologist; (5) First attack infarction/ischemic stroke. Meanwhile, infarct stroke patients with hemorrhagic transformations; history of brain tumor; history of intracranial infection; history of head trauma; aphasia; depression; and history of schizophrenia and other psychiatric disorders, were excluded.

\subsection{Data collection}

Subjects who met the inclusion criteria were underwent cognitive function examination by Neurologist, on day 5 - 7 in stroke patients with infarction in small blood vessels and day 14 in stroke patients with infarction of large blood vessels. In this study, the cognitive function was obtained from the MoCA-INA. The total score $<26$ was considered abnormal while $\geq 26$ was considered normal cognitive function. Then, the evaluation of the MSCT image head scan without contrast by a Neuroradiology consultant. Assessment focus on strategic location infarction which is hypoattenuation area of thalamus, angular gyrus, globus pallidus, basal frontal lobe, cingular gyrus, anterior crus internal capsule, caudate nucleus and hippocampus. Then, infarcts in non-strategic locations, defined as infarcts at other locations, other than strategic locations. White Matter Changes (WMC) defined as hypoattenuating areas, symmetrical, subcortical frontal, occipital, confluent periventricular to semiovale centrum. Blennow scale was used, a scale for evaluating changes in the white matter based on their degree and extent. The data obtained were then analyzed and presented as research results.

\subsection{Data analysis}

Data analysis was carried out descriptively. The variables were expressed as the absolute number and proportions $(\%)$.

\subsection{Ethical considerations}

Before this study was conducted, we requested ethical clearance from Ethical Committee of Health Research of Medical Faculty of Airlangga University and research permit from Dr. Soetomo General Hospital.

\section{Results}

Distribution of stroke infarct patients in the Diagnostic Radiology Installation of Dr. Soetomo General Hospital Surabaya for the period of January - February 2020, are stated on Table 1.

Table 1. Subject Characteristic Distributions

\begin{tabular}{lccc}
\hline & Variable & Fraquency & Percentage \\
\hline Age (years old) & $<30$ & 1 & $2 \%$ \\
& $30-40$ & 3 & $6,1 \%$ \\
& $41-50$ & 7 & $14,3 \%$ \\
& $51-60$ & 19 & $38,8 \%$ \\
& $>60$ & 19 & $38,8 \%$ \\
\hline Sex & Male & 29 & $59,2 \%$ \\
\hline
\end{tabular}




\begin{tabular}{ccc}
\hline $\begin{array}{c}\text { Infarct Location } \\
\text { Strategis }\end{array}$ & $75,5 \%$ \\
Non strategis & 12 & $24,5 \%$ \\
\hline White Matter Changes & 22 & $44,9 \%$ \\
Blennow Scale 0 - & 27 & $55,1 \%$ \\
Blennow Scale 2 - 3 & 41 & $83,7 \%$ \\
Abnormal (MoCA-INA) $<26)$ & 8 & $16,3 \%$ \\
\hline Normal (MoCA-INA) $\geq 26)$ & & \\
\hline
\end{tabular}

The results showed that most patients with infarct stroke were in the age group of 51-60 years and $>60$ years, with $38.8 \%$ (19 patients) respectively. The distribution of infarct stroke patients based on sex showed that most of the infarct stroke patients aare male, 59.2\% (29 patients). The distribution of infarct stroke patients based on location showed that there were $75.5 \%$ of infarct in strategic locations, and $24.5 \%$ of infarct in non-strategic locations. The distribution of infarct stroke patients based on white matter changes (WMC) was assessed by the Blennow scale, showing the most of them have the Blennow scale 23 , in $55.1 \%$ (27 patients). Most of the infarction stroke patients (83.7\%) have decreased cognitive function.

Infarct stroke patients who experienced cognitive dysfunction with a MoCA INA score $<26$ were mostly found in the age group $41-50$ years and $>60$ years, with $34.7 \%$ (17 patients). Stroke infarction patients who had no Cognitive function with a MoCA-INA score $\geq 26$ was mostly found in the age group $41-40$ years, $51-60$ years and $>60$ years, with $4.1 \%$ ( 2 patients).

Table 2. Cross tabulation between cognitive function and age group in stroke infarction patients at the Diagnostic Radiology Installation of Dr. Soetomo General Hospital Surabaya, during January - February 2020

\begin{tabular}{lccr}
\hline & & Cognitive Function (MoCA-INA) & Total \\
\cline { 2 - 3 } & Abnormal & Normal \\
Age Groups (years old) & & & \\
\hline$<30$ & $0(0 \%)$ & $1(2,0 \%)$ & $1(2,0 \%)$ \\
$30-40$ & $2(4,1 \%)$ & $1(2 \%)$ & $3(6,1 \%)$ \\
$41-50$ & $5(10,2 \%)$ & $2(4,1 \%)$ & $7(14,3 \%)$ \\
$51-60$ & $17(34,7 \%)$ & $2(4,1 \%)$ & $19(38,8 \%)$ \\
$>60$ & $17(34,7 \%)$ & $2(4,1 \%)$ & $19(38,8 \%)$ \\
Total & $41(83,7 \%)$ & $8(16,3 \%)$ & $49(100 \%)$ \\
\hline
\end{tabular}

Infarct stroke patients who experienced cognitive dysfunction with a MoCA INA score $<26$ were mostly found in male patients, $46.9 \%$ (23 patients). Meanwhile, stroke infarction patients who did not experience cognitive impairment (MoCA-INA score $\geq 26$ ) were mostly found in male patients, wtih $12.2 \%$ (6 patients). 
Table 3. Cross tabulation between cognitive function and sex in the Diagnostic Radiology Installation of Dr. Soetomo General Hospital Surabaya during January - February 2020

\begin{tabular}{lccc}
\hline & \multicolumn{2}{c}{ Cognitive Function (MoCA-INA) } & Total \\
\cline { 2 - 3 } & & Abnormal & Normal \\
Sex & & & \\
Male & $23(46,9 \%)$ & $6(12,2 \%)$ & $29(59,2 \%)$ \\
Female & $18(37,6 \%)$ & $2(4,1 \%)$ & $20(40,8 \%)$ \\
Total & $41(83,7 \%)$ & $8(16,3 \%)$ & $49(100 \%)$ \\
\hline
\end{tabular}

Infarct stroke patients who experienced impaired cognitive function with a MoCA INA score $<26$ were mostly found in patients with strategic infarct locations (69.4\%). Infarct stroke patients who did not experience cognitive dysfunction (MoCA-INA score $\geq 26$ ) were mostly found in the group of non-strategic infact locations $(10.2 \%)$.

Table 4. Cross tabulation between cognitive function and stroke infarction at strategic and non-strategic locations in the Diagnostic Radiology Installation of Dr. Soetomo Surabaya General Hospital for the period of January - February 2020

\begin{tabular}{|c|c|c|c|}
\hline & \multicolumn{2}{|c|}{ Cognitive Function (MoCA-INA) } & \multirow{2}{*}{ Total } \\
\hline & $\begin{array}{c}\text { Abnormal } \\
(\text { MoCA-INA <26) }\end{array}$ & $\begin{array}{c}\text { Normal } \\
(\text { MoCA-INA } \geq 26)\end{array}$ & \\
\hline \multicolumn{4}{|l|}{ Infarct Location } \\
\hline Strategic Infarct Location & $34(69,4 \%)$ & $3(6,1 \%)$ & $37(75,5 \%)$ \\
\hline Non-strategic Location & $7(14,3 \% \%)$ & $5(10,2 \%)$ & $12(24,5 \%)$ \\
\hline Total & $41(83,7 \%)$ & $8(16,3 \%)$ & $49(100 \%)$ \\
\hline
\end{tabular}

Infarct stroke patients who experienced impaired cognitive function with a MoCA INA score $<26$ were mostly found in patients with a Blennow scale $2-3(51 \%)$. Infarct stroke patients with normal cognitive function were mostly found in the Blennow 0-1 group (12.2\%).

Table 5. Cross tabulation between cognitive function and white matter changes in stroke infarct patients at the Diagnostic Radiology Installation of Dr. Soetomo General Hospital Surabaya for the period of January - February 2020

\begin{tabular}{lccc}
\hline & \multicolumn{2}{c}{ Cognitive Function (MoCA-INA) } & Total \\
\cline { 2 - 3 } & $\begin{array}{c}\text { Abnormal } \\
(\text { MoCA-INA }<26)\end{array}$ & Normal \\
(MoCA-INA $\geq 26)$ & \\
\hline Blennow Scale & $16(32,7 \%)$ & $6(12,2 \%)$ & $22(44,9 \%)$ \\
Blennow Scale 0 - & $25(51 \%)$ & $2(4,1 \%)$ & $27(55,1 \%)$ \\
Blennow Scale 2 - & $41(83,7 \%)$ & $8(16,3 \%)$ & $49(100 \%)$ \\
Total & & & $400 \%$ \\
\hline
\end{tabular}




\section{Discussion}

Impaired cognitive function have several pathophysiological mechanism, namely normal aging dementia, primary damage to gray matter (Alzheimer's disease, lobular frontotemporal degeneration, dementia with parkinsonism, dementia with other movement disorders such as Huntington's disease, drugs \& alcohol related and prion link dementia), vascular dementia/ vascular cognitive impairment, primary white matter disorders (inflammation, inborn errors of metabolism, toxic leukoencephalopathy, cognitive dysfunction associated with cancer, trauma), brain swelling dementia (giant virchow robin spaces, neoplasm and radiation necrosis, autoimmune limbic encephalitis, normal pressure hydrocephalus, Arteriovenous malformation (AVM), reversible posterior encephalopathy). ${ }^{15}$

In this study, of the 49 stroke infarction patients, $41(83.7 \%)$ had impaired cognitive function and the remaining $8(16.3 \%)$ had normal cognitive function. Infarct stroke patients who experienced cognitive impairment were mostly in the age group of 51 - 60 years, namely 17 people $(34.7 \%)$ and at the age $>60$ years, 17 people (34.7\%). This is in accordance with the literature which states that age is a non-modifiable risk factor for impaired vascular cognitive function (vascular dementia). ${ }^{16}$ As a person gets older, the volume and weight of the brain decreases, this is supported by various studies on the brain aging process which states that the brain degeneration process starts at the age of 50 years. ${ }^{17}$

Patients who experienced cognitive dysfunction, mostly (46.9\%) were male. While infarct stroke patients with normal cognitive function were found in 6 male patients $(12.2 \%)$, and 2 female patients (4.1\%). The results showed that male patients experienced more cognitive impairment than women. This is not in accordance with the literature which states that one of the risk factors for vascular cognitive impairment is female sex. ${ }^{16}$ However, research by Chaudari et al. in 2014 reported that there was no significant correlation between sex and cognitive dysfunction. ${ }^{9}$

From a total of 49 research subjects, there were 37 patients $(75.5 \%)$ with infarction at strategic locations and 12 patients $(24.5 \%)$ with infarction om non-strategic locations. Stroke patients with normal cognitive function were found in the group of strategic infarct locations $(6.1 \%)$, and 5 patients in nonstrategic infarct location (10.2\%). Stroke patients who experienced cognitive impairment were found in the group of strategic infract locations (69.4\%), and the rest in the group of non-strategic infarct locations $(14.3 \%)$. The results showed that both patients with infarction in strategic and non-strategic locations had cognitive dysfunction. This is similar to several prior studies which state that infarction at strategic locations is a predictor of cognitive dysfunction. ${ }^{3,9}$

Brain infarction can occur in strategic locations, for example the thalamus, the intravenous capsule of the anterior and genu crus, caudate nucleus, angular gyrus, globus pallidus, basal frontal lobe, cingular gyrus, bilateral medial temporo-occipital, hippocampus and watershed areas of the carotid arteries. Anatomically, the damage/ death of neurons in strategic locations can lead to the destruction of subcortical cortical circuits (thalamocortical, striatocortical and prefrontal - basal ganglia) which play important role in cognitive function. ${ }^{3}$ An ischemic-hypoxic brain parenchyma can cause dysfunction and a decrease in the number of neurons resulting in cognitive impairment. ${ }^{18}$ Impaired cognitive function also occurs in strategic locations, which can be caused by acute cerebral infarction. The literature states that $20-30 \%$ of patients 
will experience vascular dementia disorders 3 months after stroke and as many as $2 / 3$ of patients will experience cognitive impairment, if assesed in the acute stroke period. ${ }^{12,13}$

From a total of 49 subjects, there were $44.9 \%$ with no change - mild change of white matter, with the Blennow scale 0-1. Otherwise, 27 patients (55.1\%) were found in moderate to severe white matter changes, with Blennow scale 2 - 3. Patients with cognitive impairment were found in the Blennow 2- 3 scale group (51\%), and $32,7 \%$ on the Blennow scale $0-1$. Patients with normal cognitive function were found in the Blennow $0-1$ scale group of 6 people (12.2\%) and 4.1\% in the Blennow scale 2 - 3 group. The results showed that patients with a Blennow scale of 2-3 had more decline of cognitive function. This is in accordance with the literature which states that White Matter Changes (WMC) cause disruption of the white matter tract, thereby reducing the speed of transmission through the axons. Periventricular WMC is reported to cause multiple domains of cognitive impairment more frequently, because it is traversed by a substantially longer white matter tract than WMC in subcortical region, and is often associated with impaired executive function. ${ }^{3,19}$

The limitations of this study is that the type of imaging used to identify the location of the infarct and the white matter changes. MSCT scan has a lower sensitivity than MRI. In addition, cognitive function tests are carried out in the acute phase. In the acute phase, cognitive impairment may occur more frequently due to inadequate perfusion not only at the site of the lesion, but also in adjacent regions.

\section{Conclusion}

It can be concluded that cognitive function in strategic infarct locations has mostly decreased. Patients with mild, moderate to severe changes in the white matter, experience cognitive decline. Further research needs to be carried out with more variables and samples to assess the correlation between infarct location, white matter changes and other risk factors of cognitive impairment. Related to the high sensitivity of MRI in identifying changes in white matter, so studies with this modality need to be conducted.

\section{Acknowledgment}

I would like to dedicate my gratitude to all the lecturers of Radiology, Faculty of Medicine Airlangga university, my family, and my friends for their endless support and prayers.

\section{References}

Barkhof F, Fox NC, Bastos-Leite AJ. Neuroimaging in dementia. J Chem Inf Model. 2013;53(9).

Blackburn DJ, Bafadhel L, Randall M, Harkness KA. Cognitive screening in the acute stroke setting. Age Ageing. 2013;42(1):113116.

Bolandzadeh N, Davis JC, Tam R, Handy TC, et al. The association between cognitive function and white matter lesion location in older adults: A systematic review. BMC Neurol. 2012;12:1-10.

Chaudhari TS, Verma R, Garg RK, Singh MK, Malhotra HS, Sharma PK. Clinico-radiological predictors of vascular cognitive impairment (VCI) in patients with stroke: A prospective observational study. J Neurol Sci. 2014;340(1-2):150-158.

Debette S, Beiser A, Decarli C, Au R, Himali JJ, Kelly-Hayes M, et al. Association of MRI markers of vascular brain injury with incident stroke, mild cognitive impairment, dementia, and mortality: The framingham offspring study. Stroke. 2010;41(4):600606.

Dichgans M, Leys D. Vascular Cognitive Impairment. Circ Res. 2017;120(3):573-591.

García PL, García D. Diagnosis of vascular cognitive impairment and its main categories. Neurol (English Ed. 2015;30(4):223-239. Godefroy O, Fickl A, Roussel M, Auribault C, Bugnicourt JM, Lamy C, et al. Is the montreal cognitive assessment superior to the 
mini-mental state examination to detect poststroke cognitive impairment?: A study with neuropsychological evaluation. Stroke. 2011;42(6):1712-1716.

Jellinger KA. Pathology and pathogenesis of vascular cognitive impairment-a critical update. Front Aging Neurosci. 2013;5(APR):1-19.

Kalaria RN, Akinyemi R, Ihara M. Stroke injury, cognitive impairment and vascular dementia. Biochim Biophys Acta - Mol Basis Dis. 2016;1862(5):915-925.

Kandiah N, Chander RJ, Lin X, Ng A, Poh YY, Cheong CY, et al. Cognitive impairment after mild stroke: Development and validation of the SIGNAL2 risk score. J Alzheimer's Dis. 2015;49(4):1169-1177.

Kliper E, Ben Assayag E, Tarrasch R, Artzi M, Korczyn AD, Shenhar- Tsarfaty S, et al. Cognitive state following stroke: The predominant role of preexisting white matter lesions. PLoS ONE2. 2014;9(8):1-10.

Margono IS, Asriningrum, Machin A. Buku Ajar Ilmu Penyakit Saraf. eds. Machfoed MH, Hamdan M, Machin A, editors. Airlangga University Press. Surabaya; 2011

Mattson MP, Arumugam T V. Hallmarks of Brain Aging: Adaptive and Pathological Modification by Metabolic States Mark. Cell Metab. 2018;27(6):1176-99.

Ministry of Health Indonesia. Laporan RISKESDAS tahun 2013. Kementeri Kesehat RI. 2014;

Nahrowi NS, Ong PA, Adam A. Cognitive and functional outcome of patients with ischemic stroke at Dr. Hasan Sadikin hospital bandung. Althea Med J. 2018;5(2):82-86.

Simoni M, Li L, Mehta Z, Rothwell P. Prognostic value of white matter changes in patients with tia or stroke: a Population-based study. J Neurol Neurosurg Psychiatry. 2012;83(Suppl 2):A39.1-A39.

Qu Y, Zhuo L, Li N, Hu Y, Chen W, Zhou Y, et al. Prevalence of poststroke cognitive impairment in china a community-based, cross-sectional study. PLoS One. 2015;10(4):1-13.

Wattjes M, Henneman WJP, Van Der Flier WM, De Vries O, Träber F, Geurts JJG, et al. Diagnostic imaging of patients in a memory clinic: Comparison of MR imaging and 64-detector row CT. Radiology. 2009;253(1):174-183. 